\title{
On some differential inequalities in the unit disk with applications
}

Adel A Attiya*

\section{*Correspondence:}

aattiy@mans.edu.eg

Department of Mathematics,

Faculty of Science, University of

Mansoura, Mansoura, 35516, Egypt

\section{Abstract}

In this paper we obtain a number of interesting relations associated with some differential inequalities in the open unit disk, $\mathbb{U}=\{z:|z|<1\}$. Some applications of the main results are also obtained.

MSC: Primary 30C45; 30C80

Keywords: analytic functions; starlike functions; convex functions; spiral-like functions; Carathéodory functions

\section{Introduction}

Let $A$ denote the class of functions of the form

$$
f(z)=z+\sum_{n=2}^{\infty} a_{n} z^{n}
$$

which are analytic in the unit disc $\mathbb{U}=\{z:|z|<1\}$. Also, we denote by $K$ the class of functions $f(z) \in A$ that are convex in $\mathbb{U}$.

A function $f(z)$ in the class $A$ is said to be in the class $S^{*}(\alpha)$ of starlike functions of order $\alpha(0 \leq \alpha<1)$ if it satisfies

$$
\operatorname{Re}\left(\frac{z f^{\prime}(z)}{f(z)}\right)>\alpha \quad(z \in \mathbb{U})
$$

for some $\alpha(0 \leq \alpha<1)$. Also, we write $S(0)=S^{*}$, the class of starlike functions in $\mathbb{U}$.

A function $f(z) \in A$ is in $S^{\lambda}\left(|\lambda|<\frac{\pi}{2}\right)$, the class of $\lambda$-spiral-like functions, if it satisfies

$$
\operatorname{Re}\left(e^{i \lambda} \frac{z f^{\prime}(z)}{f(z)}\right)>0 \quad(z \in \mathbb{U}) .
$$

Definition 1.1 Let $f(z)$ and $F(z)$ be analytic functions. The function $f(z)$ is said to be $s u b$ ordinate to $F(z)$, written $f(z) \prec F(z)$, if there exists a function $w(z)$ analytic in $\mathbb{U}$, with $w(0)=0$ and $|w(z)| \leq 1$, and such that $f(z)=F(w(z))$. If $F(z)$ is univalent, then $f(z) \prec F(z)$ if and only if $f(0)=F(0)$ and $f(\mathbb{U}) \subset F(\mathbb{U})$.

Let $\mathbb{D}$ be the set of analytic functions $q(z)$ injective on $\overline{\mathbb{U}} \backslash E(q)$, where

$$
E(q)=\left\{\zeta \in \partial \mathbb{U}: \lim _{z \rightarrow \zeta} q(z)=\infty\right\}
$$

and $q^{\prime}(\zeta) \neq 0$ for $\zeta \in \partial \mathbb{U} \backslash E(q)$. Further, let $\mathbb{D}_{a}=\{q(z) \in \mathbb{D}: q(0)=a\}$. 
In this paper we obtain some interesting relations associated with some differential inequalities in $\mathbb{U}$. These relations extend and generalize the Carathéodory functions in $\mathbb{U}$ which have been studied by many authors e.g., see [1-14].

\section{Main results}

To prove our results, we need the following lemma due to Miller and Mocanu [15, p.24].

Lemma 2.1 Let $q(z) \in \mathbb{D}_{a}$ and let

$$
p(z)=b+b_{n} z^{n}+\cdots
$$

be analytic in $\mathbb{U}$ with $p(z) \neq b$. If $p(z) \nprec q(z)$, then there exist points $z_{0} \in \mathbb{U}$ and $\zeta_{0} \in \partial \mathbb{U} \backslash E(q)$ and on $m \geq n \geq 1$ for which

(i) $p\left(z_{0}\right)=q\left(\zeta_{0}\right)$

(ii) $z_{0} p^{\prime}\left(z_{0}\right)=m \zeta_{0} q^{\prime}\left(\zeta_{0}\right)$.

Theorem 2.1 Let

$$
P: \mathbb{U} \rightarrow \mathbb{C}
$$

with

$$
\operatorname{Re}(\bar{a} P(z))>0 \quad(a \in \mathbb{C}) .
$$

If $p$ is a function analytic in $\mathbb{U}$ with $p(0)=1$ and

$$
\operatorname{Re}\left(p(z)+P(z) z p^{\prime}(z)\right)>\frac{E}{2|a|^{2} \operatorname{Re}(\bar{a} P(z))},
$$

then

$$
\operatorname{Re}(a p(z))>\alpha,
$$

where

$$
\begin{aligned}
E= & -(\operatorname{Re}(a)-\alpha)(\operatorname{Re}(\bar{a} P(z)))^{2} \\
& +2 \operatorname{Re}(\bar{a} P(z))\left[(\operatorname{Im}(a))^{2}+2 \alpha \operatorname{Re}(a)\right] \\
& +(\operatorname{Re}(a)-\alpha)(\operatorname{Im}(a))^{2},
\end{aligned}
$$

with $\operatorname{Re}(a)>\alpha$.

Proof Let us define $q(z)$ and $h(z)$ as follows:

$$
q(z)=a p(z)
$$

and

$$
h(z)=\frac{a-(2 \alpha-\bar{a}) z}{1-z} \quad(\operatorname{Re}(a)>\alpha) .
$$


The functions $q$ and $h$ are analytic in $\mathbb{U}$ with $q(0)=h(0)=a \in \mathbb{C}$ with

$$
h(\mathbb{U})=\{w: \operatorname{Re}(w)>\alpha\} .
$$

Now, we suppose that $q(z) \nprec h(z)$. Therefore, by using Lemma 2.1, there exist points

$$
z_{0} \in \mathbb{U} \quad \text { and } \quad \zeta_{0} \in \partial \mathbb{U} \backslash\{1\}
$$

such that $q\left(z_{0}\right)=h\left(\zeta_{0}\right)$ and $z_{0} q^{\prime}\left(z_{0}\right)=m \zeta_{0} h^{\prime}\left(\zeta_{0}\right), m \geq n \geq 1$.

We note that

$$
\zeta_{0}=h^{-1}\left(q\left(z_{0}\right)\right)=\frac{q\left(z_{0}\right)-a}{q\left(z_{0}\right)-(2 \alpha-\bar{a})}
$$

and

$$
\zeta_{0} h^{\prime}\left(\zeta_{0}\right)=\frac{-\left|q\left(z_{0}\right)-a\right|^{2}}{2 \operatorname{Re}\left(a-q\left(z_{0}\right)\right)}
$$

We have $h\left(\zeta_{0}\right)=\alpha+\rho i(\alpha, \rho \in \mathbb{R})$, therefore

$$
\begin{aligned}
\operatorname{Re} & \left(p\left(z_{0}\right)+P\left(z_{0}\right) z_{0} p^{\prime}\left(z_{0}\right)\right) \\
& =\operatorname{Re}\left(\frac{1}{a} h\left(\zeta_{0}\right)+\frac{1}{a} P\left(z_{0}\right) m \zeta_{0} h^{\prime}\left(\zeta_{0}\right)\right) \\
& =\operatorname{Re}\left(\frac{\alpha+\rho i}{a}\right)-m \frac{|\alpha+\rho i-a|^{2}}{2 \operatorname{Re}(a-\alpha)} \operatorname{Re}\left(\frac{P\left(z_{0}\right)}{a}\right) \\
& \leq \operatorname{Re}\left(\frac{\alpha+\rho i}{a}\right)-\frac{|\alpha+\rho i-a|^{2}}{2 \operatorname{Re}(a-\alpha)} \operatorname{Re}\left(\frac{P\left(z_{0}\right)}{a}\right) \\
& =A \rho^{2}+B \rho+C \\
& =g(\rho),
\end{aligned}
$$

where

$$
\begin{aligned}
& A=-\frac{\operatorname{Re}\left(\bar{a} P\left(z_{0}\right)\right)}{2|a|^{2} \operatorname{Re}(a-\alpha)}, \\
& B=\frac{\operatorname{Im}(a)}{|a|^{2}}\left(1+\frac{\operatorname{Re}\left(\bar{a} P\left(z_{0}\right)\right)}{\operatorname{Re}(a)-\alpha}\right)
\end{aligned}
$$

and

$$
C=\frac{1}{|a|^{2}}\left(\alpha \operatorname{Re}(a)-\frac{\alpha^{2}+|a|^{2}-2 \alpha \operatorname{Re}(a) \operatorname{Re}\left(\bar{a} P\left(z_{0}\right)\right)}{2(\operatorname{Re}(a)-\alpha)}\right) .
$$

We can see that the function $g(\rho)$ in (2.5) takes the maximum value at $\rho_{1}$ given by

$$
\rho_{1}=\operatorname{Im}(a)\left(1+\frac{\operatorname{Re}(a)-\alpha}{\operatorname{Re}\left(\bar{a} P\left(z_{0}\right)\right)}\right) .
$$


Hence, we have

$$
\begin{aligned}
\operatorname{Re} & \left(p\left(z_{0}\right)+P\left(z_{0}\right) z p^{\prime}\left(z_{0}\right)\right) \\
& \leq g\left(\rho_{1}\right) \\
& =\frac{E}{2|a|^{2} \operatorname{Re}(\bar{a} P(z))}
\end{aligned}
$$

where $E$ is defined by (2.2). This is in contradiction to (2.1). Then we obtain $\operatorname{Re}(a p(z))>\alpha$.

Theorem 2.2 Let $p(z)$ a nonzero analytic function in $\mathbb{U}$ with $p(0)=1$. If

$$
\left|p(z)+\frac{z p^{\prime}(z)}{p(z)}-1\right|<\frac{3 \operatorname{Re}(a-\alpha)}{2|a|}|p(z)|,
$$

then

$$
\operatorname{Re}\left(\frac{a}{p(z)}\right)>\alpha
$$

where $\operatorname{Re}(a)>\alpha$.

Proof Let us define both $q(z)$ and $h(z)$ as follows:

$$
q(z)=a / p(z)
$$

and

$$
h(z)=\frac{a-(2 \alpha-\bar{a}) z}{1-z} \quad(\operatorname{Re}(a)>\alpha) .
$$

The functions $q$ and $h$ are analytic in $\mathbb{U}$ with $q(0)=h(0)=a \in \mathbb{C}$ with

$$
h(\mathbb{U})=\{w: \operatorname{Re}(w)>\alpha\} .
$$

Now, we suppose that $q(z) \nprec h(z)$. Therefore, by using Lemma 2.1, there exist points

$$
z_{0} \in \mathbb{U} \quad \text { and } \quad \zeta_{0} \in \partial \mathbb{U} \backslash\{1\}
$$

such that $q\left(z_{0}\right)=h\left(\zeta_{0}\right)$ and $z_{0} q^{\prime}\left(z_{0}\right)=m \zeta_{0} h^{\prime}\left(\zeta_{0}\right), m \geq n \geq 1$.

We note that

$$
\zeta_{0} h^{\prime}\left(\zeta_{0}\right)=\frac{-\left|q\left(z_{0}\right)-a\right|^{2}}{2 \operatorname{Re}\left(a-q\left(z_{0}\right)\right)} .
$$

We have $h\left(\zeta_{0}\right)=\alpha+\rho i(\rho \in \mathbb{R})$; therefore,

$$
\begin{aligned}
\frac{\left|p\left(z_{0}\right)+\frac{z p^{\prime}\left(z_{0}\right)}{p\left(z_{0}\right)}-1\right|}{\left|p\left(z_{0}\right)\right|} & =\left|\frac{\alpha+\rho i}{a}-\frac{m}{a} \frac{|a-\alpha-i \rho|^{2}}{2 \operatorname{Re}(a-\alpha)}-1\right| \\
& \geq \frac{1}{|a|}\left|\frac{m|a-\alpha-i \rho|^{2}}{2 \operatorname{Re}(a-\alpha)}+\operatorname{Re}(a-\alpha)\right|
\end{aligned}
$$




$$
\begin{aligned}
& \geq \frac{1}{|a|}\left(\frac{|a-\alpha-i \rho|^{2}}{2 \operatorname{Re}(a-\alpha)}+\operatorname{Re}(a-\alpha)\right) \\
& \geq \frac{1}{2|a| \operatorname{Re}(a-\alpha)}\left(3(\operatorname{Re}(a-\alpha))^{2}+(\operatorname{Im}(a)-\rho)^{2}\right) \\
& \geq \frac{3 \operatorname{Re}(a-\alpha)}{2|a|} .
\end{aligned}
$$

This is in contradiction to (2.6). Then we obtain $\operatorname{Re}\left(\frac{a}{p(z)}\right)>\alpha$.

\section{Applications and examples}

Putting $P(z)=\beta$ ( $\beta>0$; real) in Theorem 2.1 we have the following corollary.

Corollary 3.1 If $p$ is a function analytic in $\mathbb{U}$ with $p(0)=1$ and

$$
\operatorname{Re}\left(p(z)+\beta z p^{\prime}(z)\right)>\frac{E}{2 \beta|a|^{2} \operatorname{Re}(a)},
$$

then

$$
\operatorname{Re}(a p(z))>\alpha,
$$

where

$$
E=-(\operatorname{Re}(a)-\alpha) \beta^{2}(\operatorname{Re}(a))^{2}+2 \beta \operatorname{Re}(a)\left[(\operatorname{Im}(a))^{2}+2 \alpha \operatorname{Re}(a)\right]+(\operatorname{Re}(a)-\alpha)(\operatorname{Im}(a))^{2},
$$

with $\operatorname{Re}(a)>\alpha(\alpha \geq 0)$.

Putting $\beta=1$ in Corollary 3.1, we obtain the following corollary.

Corollary 3.2 If $p$ is a function analytic in $\mathbb{U}$ with $p(0)=1$ and

$$
\operatorname{Re}\left(p(z)+z p^{\prime}(z)\right)>\frac{1}{2 \operatorname{Re}(a)}(3 \operatorname{Re}(a)-\alpha)-\frac{\operatorname{Re}(a)}{|a|^{2}}(2 \operatorname{Re}(a)-3 \alpha),
$$

then

$$
\operatorname{Re}(\operatorname{ap}(z))>\alpha,
$$

with $\operatorname{Re}(a)>\alpha(\alpha \geq 0)$.

Corollary 3.3 Let $f(z) \in A,(g(z))^{a} \in S^{*}$ and

$$
\operatorname{Re}\left(\frac{f^{\prime}(z)}{g^{\prime}(z)}\right)>\frac{E}{2|a|^{2} \operatorname{Re}\left(\bar{a} \frac{g(z)}{z g^{\prime}(z)}\right)},
$$

then

$$
\operatorname{Re}\left(a \frac{f(z)}{g(z)}\right)>\alpha
$$

where $\operatorname{Re}(a)>\alpha(\alpha \geq 0)$ and $E$ is defined by (2.2) with $P(z)=\frac{g(z)}{z g^{\prime}(z)}$. 
Proof Putting $p(z)=\frac{f(z)}{g(z)}$ and $P(z)=\frac{g(z)}{z g^{\prime}(z)}$ in Theorem 2.1, we have

$$
\operatorname{Re}\left(p(z)+P(z) z p^{\prime}(z)\right)=\operatorname{Re}\left(\frac{f^{\prime}(z)}{g^{\prime}(z)}\right)
$$

Since $(g(z))^{a} \in S^{*}$, which gives $\operatorname{Re}\left(a^{z g^{\prime}(z)} \frac{g(z)}{g(z)}>0\right.$, therefore, $\operatorname{Re}(\bar{a} P(z))>0$. This completes the proof of the corollary.

Example 3.1 Let $f(z) \in A$ and

$$
\operatorname{Re}\left(f^{\prime}(z)\right)>\frac{1}{2 \operatorname{Re}(a)}(3 \operatorname{Re}(a)-\alpha)-\frac{\operatorname{Re}(a)}{|a|^{2}}(2 \operatorname{Re}(a)-3 \alpha),
$$

then

$$
\operatorname{Re}\left(a \frac{f(z)}{z}\right)>\alpha
$$

where $\operatorname{Re}(a)>\alpha$.

Example 3.2 Let $f(z) \in A$ and

$$
\operatorname{Re}\left(\left(2+\frac{z f^{\prime \prime}(z)}{f^{\prime}(z)}-\frac{z f^{\prime}(z)}{f(z)}\right) \frac{z f^{\prime}(z)}{f(z)}\right)>\frac{1}{2 \operatorname{Re}(a)}(3 \operatorname{Re}(a)-\alpha)-\frac{\operatorname{Re}(a)}{|a|^{2}}(2 \operatorname{Re}(a)-3 \alpha),
$$

then

$$
\operatorname{Re}\left(a \frac{z f^{\prime}(z)}{f(z)}\right)>\alpha
$$

where $\operatorname{Re}(a)>\alpha$.

(1) Putting $a=e^{i \lambda}\left(|\lambda|<\frac{\pi}{2}\right)$ and $\alpha=0$ in Theorem 2.1, we have Theorem 1 due to Kim and Cho [4].

(2) Putting $a=e^{i \lambda}\left(|\lambda|<\frac{\pi}{2}\right), P(z)=\beta(\beta>0$; real) and $\alpha=0$ in Theorem 2.1, we have Corollary 1 due to Kim and Cho [4].

(3) Putting $a=\alpha=0$ and $P(z)=1$ in Theorem 2.1, we have the result due to Nunokawa et al. [16].

(4) Putting $a=e^{i \lambda}\left(|\lambda|<\frac{\pi}{2}\right), P(z)=1$ and $\alpha=0$ in Theorem 2.1, we have Corollary 2 due to Kim and Cho [4].

Putting $p(z)=\frac{z f^{\prime}(z)}{f(z)}$ in Theorem 2.2, we have the following corollary.

Corollary 3.4 Let $p(z)$ a nonzero analytic function in $U$ with $p(0)=1$. If

$$
\left|\frac{z f^{\prime \prime}(z)}{f^{\prime}(z)}\right|<\frac{3 \operatorname{Re}(a-\alpha)}{2|a|}\left|\frac{z f^{\prime}(z)}{f(z)}\right|,
$$

then

$$
\operatorname{Re}\left(\frac{1}{a} \frac{z f^{\prime}(z)}{f(z)}\right)>\alpha,
$$

where $\operatorname{Re}(a)>\alpha$. 


\section{Remark}

(1) Putting $a=1$ and $\alpha=0$ in Corollary 3.4, we have the result due to Attiya and Nasr [1].

(2) Putting $a=1$ and $\alpha=0$ in Corollary 3.4, we have the result due to Kim and Cho [4].

\section{Competing interests}

The author declares that he has no competing interests.

\section{Acknowledgement}

The author would like to express his gratitude to the referee(s) for the valuable advices to improve this paper.

Received: 13 December 2013 Accepted: 6 January 2014 Published: 24 Jan 2014

\section{References}

1. Attiya, AA, Nasr, MAM: On sufficient conditions for Carathéodory functions with applications. J. Inequal. Appl. 2013, $191(2013)$

2. Cho, NE, Kim, IH: Conditions for Carathéodory functions. J. Inequal. Appl. 2009, Article ID 601597 (2009)

3. Delsarte, P, Genin, Y: A simple proof of Livingston's inequality for Carathéodory functions. Proc. Am. Math. Soc. 107. 1017-1020 (1989)

4. Kim, IH, Cho, NE: Sufficient conditions for Carathéodory functions. Comput. Math. Appl. 59(6), 2067-2073 (2010)

5. Li, JL, Owa, S: Sufficient conditions for starlikeness. Indian J. Pure Appl. Math. 33, 313-318 (2002)

6. Miller, S: Differential inequalities and Carathéodory functions. Bull. Am. Math. Soc. 81, 79-81 (1975)

7. Nunokawa, M: Differential inequalities and Carathéodory functions. Proc. Jpn. Acad., Ser. A, Math. Sci. 65, 326-328 (1989)

8. Nunokawa, M: On properties of non-Carathéodory functions. Proc. Jpn. Acad., Ser. A, Math. Sci. 68, 152-153 (1992)

9. Nunokawa, M, Owa, S, Takahashi, N, Saitoh, H: Sufficient conditions for Carathéodory functions. Indian J. Pure Appl. Math. 33, 1385-1390 (2002)

10. Obradovic, M, Owa, S: On certain properties for some classes of starlike functions. J. Math. Anal. Appl. 145, 357-364 (1990)

11. Padmanabhan, KS: On sufficient conditions for starlikeness. Indian J. Pure Appl. Math. 32, 543-550 (1990)

12. Shiraishi, H, Owa, S, Srivastava, HM: Sufficient conditions for strongly Carathéodory functions. Comput. Math. Appl. 62(8), 2978-2987 (2011)

13. Tuneski, N: On certain sufficient conditions for starlikeness. Int. J. Math. Math. Sci. 23, 521-527 (2000)

14. Yang, D, Owa, S, Ochiai, K: Sufficient conditions for Carathéodory functions. Comput. Math. Appl. 51, 467-474 (2006)

15. Miller, SS, Mocanu, PT: Differential Subordination: Theory and Application. Dekker, New York (2000)

16. Nunokawa, M, Owa, S, Nishiwaki, J, Saitoh, H: Sufficient conditions for starlikeness and convexity of analytic functions with real coefficients. Southeast Asian Bull. Math. 33(6), 1149-1155 (2009)

10.1186/1029-242X-2014-32

Cite this article as: Attiya: On some differential inequalities in the unit disk with applications. Journal of Inequalities and Applications 2014, 2014:32 